\title{
A UNIFORM DESCRIPTION OF AN OSCILLATOR'S RESONANT TRANSITION
}

\author{
P. B. CHAPMAN ${ }^{\prime}$
}

(Received 28 February 1989; revised 11 September 1989)

\begin{abstract}
A uniform approximation to the description of a linear oscillator's slow resonant transition is calculated. If the time scale of the transition is $\varepsilon^{-1}$, the approximation contains explicitly the $0(1)$ and $0\left(\varepsilon^{1 / 2}\right)$ terms, and fixes a uniform $0(\varepsilon)$ error bound.
\end{abstract}

\section{Introduction}

Linear oscillation problems on infinite and semi-infinite intervals provide a rich source of interesting and delicate problems to which there are a relatively small number of explicit solutions or substantiated approximations. Not infrequently these show unexpected properties which, even if evident in careful numerical studies, are not easily explained.

For an example of the capricious behavior of such solutions, take the asymptotic calculation of reflection coefficients [1] for the equation

$$
\varepsilon^{2} d^{2} y / d t^{2}+w^{2}(t) y=0, \quad 0<\varepsilon \ll 1 .
$$

The results are striking. The exponentially small reflection coefficient has an accumulation point of zeros as $\varepsilon \rightarrow 0, \alpha>0$ fixed, and the limits $\alpha \rightarrow 0_{+}$, $\alpha \rightarrow 0_{-}, \varepsilon$ fixed, are non-uniform, when $w(t)=\left(1+\alpha /\left(1+t^{2}\right)\right)$. This curious behaviour could not have been anticipated.

It is therefore not unreasonable to study particular oscillation problems where it is possible to obtain substantiated approximations, in order to look for significant phenomena. One can then attempt to prescribe sufficient con-

\footnotetext{
'Department of Mathematics, University of Western Australia, Nedlands, WA 6009.

(C) Copyright Australian Mathematical Society 1990, Serial-fee code 0334-2700/90
} 
ditions for similar analysis to proceed, but further computation of specific examples, and study of their results may yet be the more fruitful procedure.

A substantial literature is available in the discussion of perturbation techniques for oscillatory systems and equations with weak nonlinearities and/or slowly varying coefficients. Perhaps this literature might be categorised as modern and classical. The former category uses as a starting point the averaging method of Krylov and Bogoliubov [4] and proceeds by ingenious formal procedures to construct a succession of differential equations whose integrals provide progressively more refined approximations [3]; the latter category moves from the Liouville-Green (or equivalent) formalism [5] to Picard iterate a Volterra integral equation to a similar end. The modern approach is recently described by Kevorkian [2], while a description of the classical one is given in Olver's [5] treatise, although in a limited scenario.

The advantages of the classical approach, at least for linear problems, are that the validity proofs are directly available, a uniformly applicable approximation is naturally provided and, with that, an identification of appropriate slow and fast time scales. For example, in the problem discussed below, the uniform argument arising is

$$
\begin{aligned}
\left(\varepsilon^{-1} \ln \cosh \varepsilon t\right)^{1 / 2} & \approx(\varepsilon / 2)^{1 / 2} t & & 0 \leq \varepsilon t \ll 1 \\
& \approx(t-\ln 2 / \varepsilon)^{1 / 2} & & \varepsilon t \gg 1
\end{aligned}
$$

and the two time scales in the problem follow.

The present study examines a particular transiently-resonant linear equation

$$
d^{2} y / d t^{2}+(1+a \tanh \varepsilon t)^{2} y=k \varepsilon^{1 / 2} \sin t, \quad 0<a<1,0<\varepsilon \ll 1
$$

with two free parameters $a, \varepsilon$ (the constant $k$ can be removed by scaling, but is left in as a "tag" on terms in the calculation). It is convenient but not essential to restrict discussion to positive values of $a$; negative values can be similarly treated by reversing the sense of time. When $a=0$, equation (1.1) is resonant, and all solutions are ultimately unbounded in the limit $|t| \rightarrow \infty$, but when $a>0$ the right-hand side is only instantaneously resonant at $t=0$, where the frequency of the periodic forcing term (the right-hand side) is coincident with the natural frequency of the unforced constant-coefficient system. With a positive value of the parameter $a$, the system makes a slow (because $\varepsilon$ is small) transition through the resonance at $t=0$. For large negative $(\varepsilon t)$, it is to be expected that there will exist a solution of equation (1.1)

$$
y=(1-a)^{-1 / 2}\left(\alpha e^{i(1-a) t}+\bar{\alpha} e^{-i(1-a) t}\right)+k \varepsilon^{1 / 2} \sin t /(a(a-2))+\delta R_{-}
$$


and this could reasonably be expected to connect with a solution

$$
y=(1+a)^{-1 / 2}\left(\beta e^{i(1+a) t}+\bar{\beta} e^{i(1+a) t}\right)+k \varepsilon^{1 / 2} \sin t /(a(a+2))+\delta R_{+}
$$

at large positive $(\varepsilon t)$, where $\alpha$ and $\beta$ are constants and $\left|\delta R_{+}\right|$and $\left|\delta R_{-}\right|$ have a uniform $o\left(\varepsilon^{1 / 2}\right)$ bound. The factors $(1 \pm a)^{-1 / 2}$ are included to simplify the final statement of results, which will describe the solution of equation (1.1) for nonlarge values of $(\varepsilon t)$, establish the bound for the errors $\delta R_{ \pm}$and the relation

$$
\beta=\alpha-\left(\frac{k(1-i)}{4} \sqrt{\frac{\pi}{a}}+\frac{i k e^{1 / 2}}{4}(\sqrt{1+a}-\sqrt{1-a})\right) e^{\frac{-a \ln 2}{\varepsilon}}
$$

connecting the constants $\alpha$ and $\beta$. As Kevorkian [2] points out, the description of the transition through resonance requires a Fresnel integral, which is taken here to be

$$
F_{ \pm}(z)=\int_{0}^{z} e^{ \pm i w^{2}} d w=\int_{0}^{z} \cos w^{2} d w \pm i \int_{0}^{z} \sin w^{2} d w
$$

for a real variable $z$. Its asymptotic behaviour is

$$
\lim _{z \rightarrow \pm \infty} \int_{0}^{z} e^{ \pm i w^{2}} d w=\operatorname{sgn}(z) e^{ \pm \frac{1}{4} \pi} \sqrt{\frac{\pi}{2}}=\operatorname{sgn}(z)\left(\frac{1 \pm i}{2}\right) \sqrt{\frac{\pi}{2}}
$$

\section{Analysis}

The equation

$$
d y^{2} / d t^{2}+(1+a \tanh u)^{2} y=k \varepsilon^{1 / 2} \sin t
$$

is studied as a model of a transiently resonant system, where

$$
u=\varepsilon t
$$

$k, a(0<a<1)$, and $\varepsilon(0<\varepsilon \ll 1)$ are real parameters. The coefficient

$$
(1+a \tanh u)^{2} \equiv(d \phi / d u)^{2}
$$

is slowly varying between $(1-a)^{2}$ and $(1+a)^{2}$ as $u \rightarrow-\infty$, and $+\infty$, respectively and at $u, t=0$ the right-hand side is instantaneously resonant. The definition of $d \phi / d u$ allows a choice

$$
\phi(u)=u+a \ln (\cosh u)
$$

and requires

$$
d^{2} \phi / d u^{2}=a \operatorname{sech}^{2} u
$$


The boundedness and decay behaviour of the second derivative (2.5)

$$
\operatorname{sech}^{2} u \sim e^{-2|u|} / 4, \quad|u| \rightarrow \infty
$$

confer desirable properties on integrands which arise in the construction of the solution of (2.1).

It might reasonably be supposed that a solution of $(2.1)$ which behaves as

$$
y=(1-a)^{-1 / 2}\left(\alpha e^{i(1-a) t}+\bar{\alpha} e^{-t(1-a) t}\right)+k \varepsilon^{1 / 2} \sin t /(a(a-2))
$$

as $(\varepsilon t) \rightarrow-\infty$, will behave as

$$
y=(1+a)^{-1 / 2}\left(\beta e^{i(1+a) t}+\bar{\beta} e^{-i(1+a) t}\right)+k \varepsilon^{1 / 2} \sin t /(a(a+2))+\delta R
$$

as $\varepsilon t \rightarrow \infty$, where $\delta$, a constant depending on $\varepsilon$, is $o\left(\varepsilon^{1 / 2}\right)$ and $R$ is uniformly bounded. The aim of the present study is to calculate $y$ when $(\varepsilon t)$ is not large, and to establish the $\beta(\alpha)$ and $\delta(\varepsilon)$ relationships. In doing this, it is convenient to integrate from the origin $t=0$ both backwards and forwards but, in order to suppress the irrelevant complication of the presence of $\operatorname{sgn}(u)$ factors in expressions, only the details of the forward integration are given; those of the backward integration are analogous.

Following the Liouville-Green approximation [5], a solution of equation (2.1) is expressed as

$$
y=W e^{\frac{i \phi}{e}} / \sqrt{\phi^{\prime}}+\bar{W} e^{\frac{-i \phi}{e}} / \sqrt{\phi^{\prime}}
$$

(where the convention ' $=\frac{d}{d u}$ is employed), with the condition

$$
\left(W / \sqrt{\phi^{\prime}}\right)^{\prime} e^{\frac{i \phi}{e}}+\left(\bar{W} / \sqrt{\phi^{\prime}}\right)^{\prime} e^{\frac{-i \phi}{e}}=0
$$

imposed as one of the two necessary to determine the complex valued function $W$ of the real variable $u$. The second condition is that the $y$ so defined (by (2.8)) satisfies the differential equation (2.1), and this can be shown to be

$$
W^{\prime}=-k \varepsilon\left(e^{\frac{u t}{e}}-e^{\frac{-1 u}{e}}\right) e^{\frac{-1 \phi}{e}} /\left(4 \sqrt{\phi^{\prime}}\right)+\bar{W} \phi^{\prime \prime} e^{\frac{-2 i \phi}{e}} /\left(2 \phi^{\prime}\right)
$$

after using (2.9).

Still following, for example, Olver's [4] proof of the Liouville-Green approximation, $(2.10)$ is restated as a Volterra integral equation

$$
\begin{aligned}
W(u) & =W(0)-\frac{k \varepsilon^{-1 / 2}}{4} \int_{0}^{u}\left(e^{\frac{u(v-\phi)}{e}}-e^{\frac{-u(v+\phi)}{e}}\right) \frac{d v}{\sqrt{\phi^{\prime}}}+\int_{0}^{u} \frac{\bar{W} \phi^{\prime \prime}}{2 \phi^{\prime}} e^{\frac{-2 i \phi}{e}} d v \\
& \equiv W(0)-\frac{k \varepsilon^{-1 / 2}}{4}\left(J_{1}(u)-J_{2}(u)\right)+\int_{0}^{u} \frac{\bar{W} \phi^{\prime \prime}}{2 \phi^{\prime}} e^{\frac{-21 \phi}{e}} d v \\
& \equiv W_{0}(u)+\int_{0}^{u} \frac{\bar{W} \phi^{\prime \prime}}{2 \phi^{\prime}} e^{\frac{-2 l \phi}{\varepsilon}} d v
\end{aligned}
$$


where $W_{0}(u), J_{1}(u)$ and $J_{2}(u)$ are defined in a natural way. Solutions of this integral equation (2.11) are differentiable, and will satisfy the differential equation (2.10) on their existence interval.

The existence and uniqueness of solutions of the integral equation (2.11) is proved by showing convergence of its Picard iteration

$$
W_{n+1}(u)-W_{n}(u)=\int_{0}^{u}\left(\bar{W}_{n}(v)-\bar{W}_{n-1}(v)\right) \frac{\phi^{\prime \prime}}{2 \phi^{\prime}} e^{\frac{-2 u \phi}{\varepsilon}} d v
$$

$n=0,1, \ldots$ with $W_{-1}(u) \equiv 0$ on some interval $[O, U]$ where $U \geq 0$. If, for the existence interval $[0, U]$, (which will later be shown to be $[0, \infty)$ ) a constant $d$ can be chosen so that

$$
\left|W_{1}(u)-W_{0}(u)\right| \leq d \ln \sqrt{\phi^{\prime}}
$$

then for any $u$ in the interval

$$
\left|W_{2}-W_{1}\right| \leq d \int_{0}^{u} \ln \left(\sqrt{\phi^{\prime}}\right) \frac{\phi^{\prime \prime}}{2 \phi^{\prime}} d v
$$

since both $\phi^{\prime \prime}$ and $\phi^{\prime}$ are positive $(2.3,2.5)$. Thus it follows that

$$
\left|W_{2}-W_{1}\right| \leq d\left(\ln \sqrt{\phi^{\prime}}\right)^{2} / 2
$$

and by induction,

$$
\left|W_{n}-W_{n-1}\right| \leq d\left(\ln \sqrt{\phi^{\prime}}\right)^{n} / n !
$$

Thus the sequence $\left\{W_{n}\right\}$ is pointwise convergent on $[0, U]$, and uniformly convergent, by the $M$ test. For a continuous $W_{0}$, the successive Picard iterates are continuous functions of $u$, so their (unique) $\lim _{n \rightarrow \infty} W_{n}=W$ is a continuous function and satisfies

$$
\left|W-W_{0}\right| \leq d\left(\sqrt{\phi^{\prime}}-1\right) .
$$

It is now routine to show that the limit satisfies the integral equation (2.11) on any interval $0 \leq u$ (i.e. $U$ is unbounded) and thus the differential equation (2.10), so it only remains to calculate $W_{0}$, and show that $d$ is sufficiently small, that approximating $W$ by $W_{0}$ is useful. An approximation evaluation of $W_{0}$ is first given.

In Appendix A1 the integral $J_{1}(u)=\int_{0}^{u} e^{\frac{(t v-\phi)}{e}} / \sqrt{\phi^{\prime}} d v$ is calculated and it 
is shown that, in terms of the Fresnel integral (1.5)

$$
\begin{aligned}
J_{1}(u) & =(2 \varepsilon / a)^{1 / 2} F_{-}(\sqrt{a \ln \cosh u / \varepsilon}) \\
& +\frac{\varepsilon i}{a}\left\{e^{\frac{-i a \ln \cosh u}{\epsilon}}\left((\tanh u)^{-1}(1+a \tanh u)^{-1 / 2}-(2 \ln \cosh u)^{-1 / 2}\right)+\frac{a}{2}\right\} \\
& +\varepsilon^{3 / 2} \sigma_{1} \quad u>0
\end{aligned}
$$

where $\left|\sigma_{1}\right|<s_{1}$ and $s_{1}$ is a constant, independent of $\varepsilon$.

In Appendix A2 the integral $J_{2}(u)=\int_{0}^{u} e^{\frac{-(t u+\phi)}{\varepsilon}} / \sqrt{\phi^{\prime}} d v$ is calculated, and it is shown that

$$
J_{2}(u)=\varepsilon i\left[\frac{e^{\frac{-\imath(u+\phi)}{\varepsilon}}}{\left(1+\phi^{\prime}\right) \sqrt{\phi^{\prime}}}-\frac{1}{2}\right]+\varepsilon^{2} \sigma_{2} \quad u>0
$$

where $\left|\sigma_{2}\right|<s_{2}$ and $s_{2}$ is a constant, independent of $\varepsilon$. It then follows that the uniformly applicable approximation to

$$
W_{0}(u)=W(0)-k \varepsilon^{-1 / 2}\left(J_{1}(u)-J_{2}(u)\right) / 4
$$

is

$$
\begin{array}{r}
W_{0}(u)=W(0)-\frac{k}{4}\left(\left(\frac{2}{a}\right)^{1 / 2} F_{-}\left(\sqrt{\frac{a \ln \cosh u}{\varepsilon}}\right)-\varepsilon^{1 / 2} i\left[\frac{e^{\frac{-t(u+\phi)}{\varepsilon}}}{\left(1+\phi^{\prime}\right) \sqrt{\phi^{\prime}}}-\frac{1}{2}\right]\right. \\
+\frac{\varepsilon^{1 / 2} i}{a}\left\{e ^ { \frac { - t a \operatorname { l n } \operatorname { c o s h } u } { \varepsilon } } \left((\tanh u)^{-1}(1+a \tanh u)^{-1 / 2}\right.\right. \\
\left.\left.\left.-(2 \ln \cosh u)^{-1 / 2}\right)+\frac{a}{2}\right\}+O(\varepsilon)\right) .
\end{array}
$$

Here we note, that as $u \rightarrow \infty \quad(\ln \cosh u \rightarrow u-\ln 2, \phi \rightarrow u(1+a)-a \ln 2)$

$$
\begin{aligned}
& W_{0}(u) \rightarrow W(0)-\frac{k}{4}\left(\frac{(1-i)}{2} \sqrt{\frac{\pi}{4}}-\varepsilon^{1 / 2} i\left[\frac{e^{\frac{-i(u+\phi)}{e}}}{(2+a)(1+a)^{1 / 2}}-\frac{1}{2}\right]\right.
\end{aligned}
$$

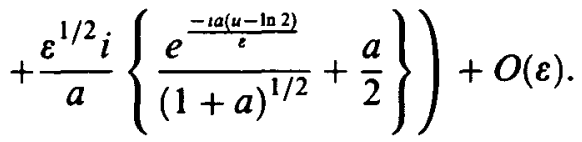


Hence also follows the limit behaviour

$$
\begin{aligned}
\frac{W_{0} e^{\frac{\phi \phi}{\varepsilon}}}{\sqrt{\phi^{\prime}}} \rightarrow & \left(\frac{W(0)}{(1+a)^{1 / 2}}-\frac{k(1-i)}{8(1+a)^{1 / 2}} \sqrt{\frac{\pi}{a}}-\frac{k \varepsilon^{1 / 2} i}{4}\right) e^{\frac{i u(1+a)-1 a \ln 2}{e}} \\
& +\frac{k \varepsilon^{1 / 2} i}{4}\left[\frac{e^{\frac{-i u}{e}}}{(2+a)(1+a)}-\frac{e^{\frac{u}{\varepsilon}}}{a(1+a)}\right]+O(\varepsilon)
\end{aligned}
$$

and the contribution to the solution $y$ from the first term above is

$$
2 R 1\left\{\frac{k \varepsilon^{1 / 2} i}{4}\left[\frac{e^{\frac{-i u}{e}}}{(2+a)(1+a)}-\frac{e^{\frac{u u}{e}}}{a(1+a)}\right]\right\}=k \varepsilon^{1 / 2} \frac{\sin \left(\frac{u}{\varepsilon}\right)}{a(2+a)}
$$

as was anticipated from (2.7). Similarly, as $u \rightarrow \infty$, analogous computations show that

$$
\begin{aligned}
\frac{W_{0} e^{\frac{i \phi}{e}}}{\sqrt{\phi^{\prime}}} \rightarrow\left(\frac{W(0)}{(1-a)^{1 / 2}}\right. & \left.+\frac{k(1-i)}{8(1-a)^{1 / 2}} \sqrt{\frac{\pi}{a}}-\frac{k \varepsilon^{1 / 2} i}{4}\right) e^{\frac{i u(1+a)-i a \ln 2}{e}} \\
& +\frac{k \varepsilon^{1 / 2} i}{4}\left[\frac{e^{\frac{i u}{e}}}{a(1-a)}+\frac{e^{\frac{-i u}{e}}}{(2+a)(1-a)}\right]
\end{aligned}
$$

and the second term here contributes $\frac{k \varepsilon^{1 / 2} \sin \left(\frac{u}{e}\right)}{a(a-2)}$ to the solution $y$, as anticipated from (2.6).

The $\beta(\alpha)$ relationship is therefore obtained by eliminating $W(0)$ between appropriate parts of the expressions (2.19) and (2.20). It is

$$
\beta=\alpha-\left(\frac{k(1-i)}{4} \sqrt{\frac{\pi}{a}}+\frac{i k \varepsilon^{1 / 2}}{4}(\sqrt{1+a}-\sqrt{1-a})\right) e^{\frac{-i a \ln 2}{i}}
$$

The calculation of (c.f. (2.11), (2.13)).

$$
D(u)=W_{1}(u)-W_{0}(u)=\int_{0}^{u} \frac{W_{0} \phi^{\prime \prime}}{2 \phi^{\prime}} e^{\frac{-2 l \phi}{e}} d v
$$

or, more specifically, the constant $d$ in the bound $|D(u)| \leq d \ln \sqrt{\phi^{\prime}}$ follows from the evaluation of

$$
Z(u)=\frac{1}{\varepsilon} \int_{0}^{u} \frac{\phi^{\prime \prime}}{2 \phi^{\prime}} e^{\frac{-2 i \phi}{\varepsilon}} d v=c+\frac{i \phi^{\prime \prime}}{4\left(\phi^{\prime}\right)^{2}} e^{\frac{-2 i \phi}{e}}+\sigma_{3}
$$

(where $c \approx-i a / 4$ is an $0(1)$ complex constant and $\left|\sigma_{3}\right| \leq \varepsilon L e^{-2 u}$ with $L$ an $O(1)$ constant), which is shown in the appendix $A 3$. 
With this definition (2.23) of $Z$, one has

$$
\begin{aligned}
D(u) & =\varepsilon \int_{0}^{u} Z^{\prime} \bar{W}_{0} d v \\
& =\varepsilon\left[Z \varepsilon W_{0}\right]_{0}^{u}-\varepsilon \int_{0}^{u} \overline{W_{0}^{\prime}} Z d v
\end{aligned}
$$

and, (from 2.10, 2.11) $W_{0}^{\prime}$ is known and

$$
{\overline{W^{\prime}}}_{0}=-k \varepsilon^{-1 / 2}\left(e^{\frac{-i(u-\phi)}{\varepsilon}}-e^{\frac{+((u+\phi)}{e}}\right) /\left(4 \sqrt{\phi^{\prime}}\right),
$$

so, using (2.23), obtain an integral expression

$$
\begin{aligned}
D(u)=\varepsilon\left[Z \bar{W}_{0}\right]_{0}^{u}-\frac{k \varepsilon^{1 / 2}}{4} & \int_{0}^{u}\left(e^{\frac{-i(v-\phi)}{e}}-e^{\frac{i(v+\phi)}{e}}\right) . \\
& \left(c+\frac{i e^{\frac{-21 \phi}{e}} \phi^{\prime \prime}}{4\left(\phi^{\prime}\right)^{2}}+\sigma_{3}\right) \frac{d v}{\sqrt{\phi^{\prime}}}
\end{aligned}
$$

which can be estimated, as follows.

The integrated part $\varepsilon[]_{0}^{u}$ has a uniform $O(\varepsilon)$ bound on the positive half line, from the bounds on $Z$ and $W_{0}$. The integral

$$
k \varepsilon^{1 / 2} \int_{0}^{u}\left(e^{\frac{-t(v-\phi)}{e}}-e^{\frac{t(v+\phi)}{e}}\right)\left(c+\sigma_{3}\right) / \sqrt{\phi^{\prime}} d v
$$

is subject to a uniform $0(\varepsilon)$ bound $(u \geq 0)$, the dominant part being evaluated as in A1. The integral $k \varepsilon^{1 / 2} \int_{0}^{u} e^{\frac{-u(v+\phi)}{\epsilon}} \phi^{\prime \prime} /\left(4\left(\phi^{\prime}\right)^{5 / 2}\right) d v$ can be treated as in $\mathrm{A} 2$, and a uniform $0\left(\varepsilon^{3 / 2}\right)$ bound established for $u \geq 0$. There remains the integral in $(2.25)$ to be bounded:

$$
\begin{aligned}
T(u) & \equiv k \varepsilon^{1 / 2} \int_{0}^{u} e^{\frac{-t(v-\phi)}{\varepsilon}} \frac{\phi^{\prime \prime}}{\left(\phi^{\prime}\right)^{5 / 2}} d v \\
& =k \varepsilon^{1 / 2} \int_{0}^{u} \bar{J}_{1}^{\prime}(v)\left(\frac{\phi^{\prime \prime}}{\left(\phi^{\prime}\right)^{5 / 2}}-a\right) d v+k a \varepsilon^{1 / 2} \bar{J}_{1}(u)
\end{aligned}
$$

where the right hand side is obtained using the definition of (A1.1) of $J_{1}$ and using

$$
\phi^{\prime \prime} /\left.\left(\phi^{\prime}\right)^{5 / 2}\right|_{v=0}=a .
$$

The total variation $V$ of $\left(\frac{\phi^{\prime \prime}}{\left(\phi^{\prime}\right)^{3 / 2}}-a\right)$ is finite on the positive half line, so ([2], p. 51)

$$
\begin{aligned}
& \left|\varepsilon^{1 / 2} \int_{0}^{u} \bar{J}_{1}^{\prime}(v)\left(\frac{\phi^{\prime \prime}}{\left(\phi^{\prime}\right)^{5 / 2}}-a\right) d v\right| \\
& \quad \leq \varepsilon^{1 / 2}\left(\max _{(0, \infty)} \frac{\left.\operatorname{Im} \bar{J}_{1}-\min _{1} \operatorname{Im} \bar{J}_{1}\right) v \sqrt{2},}{R l \bar{J}_{1}}\right)
\end{aligned}
$$


and since the real and imaginary parts of $J_{1}$ have an $0\left(\varepsilon^{1 / 2}\right)$ bound for $u \geq 0$ (A1.2), then $|T(u)|$ has an $0(\varepsilon)$ bound. Hence, finally there exists a constant $M$ such that $|D(u)| \leq M \varepsilon$ for $u \geq 0$ so

$$
d=M \varepsilon
$$

or, in (1.3) $\left|\delta R_{+}\right|$is $0(\varepsilon)$ uniformly, and similarly $\left|\delta R_{-}\right|$, that is $\delta=\varepsilon$.

\section{Concluding remarks}

The transition through resonance of the more general oscillator

$$
\varepsilon^{2} d^{2} y / d t^{2}+(1+\Omega(u))^{2} y=k \varepsilon^{1 / 2} \sin t
$$

can be described using the same analytic approach provided the real valued function

$$
\Omega(u)=\phi^{\prime}(u)-1
$$

is suitably restricted. Examination of the preceding calculations suggest that the following restrictions suffice for any twice continuously differentiable $\Omega$.

(1) $1+\Omega(u) \geq \delta>0$ for all $u$, where $\delta$ is independent of $\varepsilon$;

(2) $\lim _{u \rightarrow \pm \infty}(\Omega(u))=k_{ \pm}$where $k_{ \pm}$are positive constants;

(3) $\Omega(u)=0$ has a unique solution $u=0$;

(4) $\Omega^{\prime}(0) \neq 0$;

(5) $\Omega^{\prime \prime}(u)=\phi^{\prime \prime \prime}(u)$ is continuous and both locally and improperly integrable; and

(6) $\left|\Omega^{\prime}(u)\right|,\left|u \Omega^{\prime \prime}(u)\right|$, (and hence $\left.\left|\Omega^{\prime \prime}(u)\right|\right)$ are both locally and improperly integrable

Restrictions (1) and (2) validate the algebra used to pose the problem and (3) and (4) ensure that there is one transition point, and that solutions are locally described by the Fresnel integrals $F_{ \pm}$. Restrictions (5) and (6) are necessary to make the error estimates; in particular (6) makes the total variation of $\phi^{\prime \prime}$ and $\phi^{\prime \prime \prime}$ finite on any interval of $u$. Thus the analysis of the previous section can be followed through with a much slower decay of $\Omega$ to its asymptotic values-for example algebraic decay

$$
\left.\begin{array}{l}
\Omega(u)=\alpha /\left(1+u^{2}\right) \\
\phi(u)=u+\alpha \tan ^{-1} u
\end{array}\right\} \quad \alpha>0
$$

or

$$
\left.\begin{array}{l}
\Omega(u)=\alpha \tan ^{-1} u \\
\phi(u)=u+\alpha\left(u \tan ^{-1} u-\ln \left(1+u^{2}\right) / 2\right)
\end{array}\right\} \quad|\alpha|<\frac{2}{\pi} .
$$


In retrospect, the calculated example's exponential asymptotic decay rate (in Section 2) is something of an overkill.

The key to the docility of integral estimates in the convergence proof to the success of the method, lies in the presence of the $\phi^{\prime \prime}$ factor in the residual in $(2.10)$

$$
\left(W^{\prime}-W_{0}^{\prime}\right)=\bar{W} \phi^{\prime \prime} e^{-\frac{2 t \phi}{e}} /\left(2 \phi^{\prime}\right)
$$

with its attendant asymptotic decay properties, as can be seen from the proof of the Picard iteration's convergence (Section 2). The damped, nonlinearly perturbed form of (3.1), viz.

$$
\frac{d^{2} y}{d t^{2}}+2 \beta \varepsilon \frac{d y}{d t}+(1+\Omega)^{2} y=k \varepsilon^{1 / 2} \sin t+\delta f\left(y, \frac{d y}{d t}\right) \quad \beta>0
$$

would generate an equation (corresponding to 2.10)

$$
\begin{aligned}
W^{\prime}+\beta W= & W_{0}^{\prime}+\bar{W} e^{-\frac{2 l \phi}{e}}\left(\frac{\phi^{\prime \prime}}{2 \phi^{\prime}}+\beta\right) \\
& -\frac{i \delta e^{\frac{-i \phi}{e}}}{2 \sqrt{\phi^{\prime} \varepsilon}} f\left(\frac{W e^{\frac{i \phi}{e}}+\bar{W} e^{\frac{-i \phi}{e}}}{\sqrt{\phi^{\prime}}}, i \sqrt{\phi^{\prime}}\left(W e^{\frac{i \phi}{e}}-\bar{W} e^{\frac{-i \phi}{e}}\right)\right)
\end{aligned}
$$

where $W_{0}$ is specified in (2.11).

The absence of the mollifying factor $\phi^{\prime \prime}$ from part of the residual $\left(W^{\prime}+\right.$ $\beta W-W_{0}^{\prime}$ ) will restrict the interval of applicability of convergence proofs, and make consequent estimates less controllable, for any nonzero $\delta$. Then a solution matching procedure may be inevitable, as in [2]. If $\delta=0$, then the Picard iteration which is natural,

$$
W_{n+1}(u)-W_{n}(u)=e^{-\beta u} \int_{0}^{u}\left(\phi^{\prime \prime} /\left(2 \phi^{\prime}\right)+\beta\right) e^{\frac{-2 i \phi}{i}}\left(\bar{W}_{n}-\bar{W}_{n-1}\right) d W
$$

converges provided

$$
\int_{0}^{u}\left|\phi^{\prime \prime} /\left(2 \phi^{\prime}\right)\right| d u=S(u)
$$

is uniformly bounded for all $u>0$. This follows on defining:

$$
\mathscr{S}(u)=\int_{0}^{u}\left(\left|\phi^{\prime \prime} /\left(2 \phi^{\prime}\right)\right|+\beta\right) d w
$$

and making the inductive hypothesis

$$
\left|W_{n}(u)-W_{n-1}(u)\right| \leq e^{-\beta u} \mathscr{S}^{n}(u) d / n !
$$

It follows that

$$
\left|W_{n+1}(u)-W_{n}(u)\right| \leq e^{-\beta u} \mathscr{S}^{n+1}(u) d /(n+1) !,
$$


so that the sequence $\left\{W_{n}(u)\right\}$ converges for any $u$, and

$$
\begin{aligned}
\left|W(u)-W_{0}(u)\right| & \leq d\{\exp (\mathscr{S}(u)-\beta u)-1\} \\
& =d\{\exp (\mathscr{S}(u))-1\}
\end{aligned}
$$

where $W_{0}(u)=e^{-\beta u} \int_{0}^{u} e^{\beta w} W_{0}^{\prime} d w$ and

$$
\alpha=\left\|W_{1}-W_{0}\right\| \text {. }
$$

The condition (3.7) is exactly the same as that for the convergence of the undamped, linear system; this indifference to damping is perhaps to be expected.

\section{Appendix 1}

The integral defining the function

$$
J_{1}(u)=\int_{0}^{u} e^{\frac{i(v-\phi)}{\varepsilon}} / \sqrt{\phi^{\prime}} d v
$$

where $\phi$ is defined in (2.4), is reduced by the change of variable $v(w)=$ $\cosh ^{-1}\left(e^{\frac{w^{2}}{2}}\right)(w(v)=\sqrt{2 \ln \cosh v})$ to

$$
J_{1}(u)=\int_{0}^{w(u)} e^{-\frac{-a w^{2}}{2 e}} w\left(1-e^{-w^{2}}\right)^{-1 / 2}\left(1+a\left(1-e^{-w^{2}}\right)^{1 / 2}\right)^{-1 / 2} d w
$$

The latter's integrand possesses a limit at $w=0$, which allows writing it as

$$
\begin{aligned}
J_{1}(u)= & \int_{0}^{w(u)} e^{\frac{-a a w^{2}}{2} \varepsilon} d w \\
& +\int_{0}^{w(u)} e^{\frac{-a a w^{2}}{2} \varepsilon}\left[\left(1-e^{-w^{2}}\right)^{-1 / 2}\left(1+a\left(1-e^{-w^{2}}\right)^{1 / 2}\right)^{-1 / 2}-w^{-1}\right] d w
\end{aligned}
$$

The first of these integrals is a complex Fresnel integral $\left(\frac{2 e}{a}\right)^{1 / 2} F_{-}\left(\left(\frac{a}{2 \varepsilon}\right)^{1 / 2} w(u)\right)$, where $F_{-}$is defined in (1.5). The factor [ ] in the second (together with its limit $-\frac{a}{2}$ at $w=0$ ) defines an analytic function on the real line, which decays as $w \rightarrow \infty$ to a constant []$=(1+a)^{-1 / 2}-w^{-1}+0\left(e^{-w^{2}}\right)$. Thus an integration by parts yields

$$
\begin{array}{r}
\int_{0}^{w(u)} w e^{\frac{-\imath a w^{2}}{2} \varepsilon}[] d w=\frac{i \varepsilon}{a}\left\{\left(\left.e^{\frac{-i a w^{2}}{2} \varepsilon}[]\right|_{w=w(u)}+\frac{a}{2}\right)\right. \\
\left.-\int_{0}^{w(u)} e^{\frac{-i a w^{2}}{2} e} \frac{d}{d w}[] d w\right\} .
\end{array}
$$


The factor in the last integrand $\frac{d}{d w}[]$ is analytic on the real line, and decays as $w \rightarrow \infty$ as $0\left(w^{-2}\right)$. Its total variation $V$ on the interval $[0, \infty)$ is finite, and so

$$
\int_{0}^{w(u)} e^{\frac{-1 a w^{2}}{2} \varepsilon} \frac{d}{d w}[] d w=\left(\frac{2 \varepsilon}{a}\right)^{1 / 2}\left(\left.\frac{d}{d w}[]\right|_{w=0}\right) F_{-}\left(\left(\frac{1}{2 \varepsilon}\right)^{1 / 2} w(u)\right)+M
$$

where

$$
|M| \leq\left(\frac{2 \varepsilon}{a}\right)^{1 / 2} \sqrt{\pi} V,
$$

by the second mean value theorem for integrals ([2] p. 51) since

$$
\max _{0 \leq w} \int_{0}^{2} \cos w^{2} d w-\min \int_{0}^{w} \cos w^{2} d w \leq \sqrt{\frac{\pi}{2}}
$$

Then, in summary, we have

$$
\begin{aligned}
& J_{1}(u)=\left(\frac{2 \varepsilon}{a}\right)^{1 / 2} F_{-}\left(\left(\frac{a}{2 \varepsilon}\right)^{1 / 2} w(u)\right) \\
& +\frac{i \varepsilon}{a}\left\{\left.e^{\frac{-t a w^{2}}{2} \varepsilon}\left[\left(1-e^{-w^{2}}\right)^{-1 / 2}\left(1+a\left(1-e^{-w^{2}}\right)^{1 / 2}\right)^{-1 / 2}-w^{-1}\right]\right|_{w=w(u)}+\frac{a}{2}\right\} \\
& +\varepsilon^{3 / 2} \sigma_{1}
\end{aligned}
$$

where $\left|\sigma_{1}\right|$ is uniformly bounded, independent of $\varepsilon,\left|\sigma_{1}\right|<A_{1}$.

\section{Appendix 2}

The evaluation of the integral

$$
J_{2}(u)=\int_{0}^{u} e^{\frac{-t(v+\phi)}{e}} / \sqrt{\phi^{\prime}} d v
$$

where $\phi$ is defined in equation (2.4), is by partial integration and error estimation. The partial integration is possible since the derivative

$$
\frac{d}{d v}(v+\phi)=2+a \tanh v
$$

vanishes nowhere on $[0, \infty)$ (or on $(-\infty, 0]$, as $0<a<1$ ).

Thus the integral is

$$
\begin{aligned}
J_{2}(u)= & i \varepsilon\left[\left.\left(\frac{e^{\frac{-u(v+\phi)}{e}}}{\left(1+\phi^{\prime}\right) \sqrt{\phi^{\prime}}}\right)\right|_{v=u}-\frac{1}{2}\right] \\
& -i \varepsilon \int_{0}^{u} e^{\frac{-u(v+\phi)}{\varepsilon}} \frac{d}{d v}\left(\frac{1}{\left(1+\phi^{\prime}\right) \sqrt{\phi^{\prime}}}\right) d v
\end{aligned}
$$


with the last term being taken as the error. Rewrite the error integral as

$$
E=\int_{0}^{u} \frac{e^{\frac{-i(v+\phi)}{c}}}{\left(1+\phi^{\prime}\right)}\left\{\left(1+\phi^{\prime}\right) \frac{d}{d v}\left(\frac{1}{\left(1+\phi^{\prime}\right)} \sqrt{\phi^{\prime}}\right)\right\} d v
$$

and apply the second mean value theorem ([1] p. 51). The factor \{\} in the integrand of the expression for $E$ itself contains a factor $\phi^{\prime \prime}(v)=\operatorname{sech}^{2} v$ and therefore decays exponentially as $|v| \rightarrow \infty$; and defining the total variation of $\left.\{\}\right|_{v \rightarrow \infty}-\left.\{\}\right|_{v=0}$ to be $V_{1}$, we have

$$
E=\left.\{\}\right|_{v=0} i \varepsilon\left(e^{\frac{u(v+\phi)}{e}}-1\right)+E_{1}
$$

with $\left|E_{1}\right|<\varepsilon V_{1} 2 \sqrt{2}$. Hence it follows that $|E| \leq s_{2}$ where the constant $\sigma_{2}$ can be chosen independently of $\varepsilon$.

\section{Appendix 3}

Since $\phi^{\prime \prime}=a \operatorname{sech}^{2} u, \phi^{\prime}=1+\tanh u$, the factor $\frac{\phi^{\prime \prime}}{\phi^{\prime}}$ in the integrand in the definition of

$$
Z(u)=\frac{1}{\varepsilon} \int_{0}^{u} \frac{\phi^{\prime \prime}}{2 \phi^{\prime}} e^{\frac{-2 i \phi}{\varepsilon}} d v
$$

is bounded and exponentially decaying $\left(0\left(e^{-2|u|}\right)\right)$ as $u \rightarrow \infty$. Then integration by parts shows that

$$
\begin{aligned}
Z(u)=\left[i \frac{\phi^{\prime \prime}}{4\left(\phi^{\prime}\right)^{2}} e^{\frac{-2 i \phi}{\varepsilon}}\right]_{v=0}^{u} & +\left[\frac{\varepsilon}{8 \phi^{\prime}} \frac{d}{d u}\left(\frac{\phi^{\prime \prime}}{\left(\phi^{\prime}\right)^{2}}\right) e^{\frac{-2 i \phi}{\epsilon}}\right]_{v=0}^{u} \\
& -\frac{\varepsilon}{8} \int_{0}^{u} \frac{d}{d u}\left(\frac{1}{\phi^{\prime}} \frac{d}{d u}\left(\frac{\phi^{\prime \prime}}{\left(\phi^{\prime}\right)^{2}}\right)\right) e^{\frac{-2 i \phi}{\varepsilon}} d u
\end{aligned}
$$

Because $(-) \frac{d}{d u}\left(\frac{\phi^{\prime \prime}}{\left(\phi^{\prime}\right)^{2}}\right)$ is positive, and monotone decreasing to zero at $u \rightarrow$ $\infty$, and $\left(\phi^{\prime}\right)^{-1}$ is positive and monotone decreasing to its constant value $(1+a)^{-1}$ in the same limit, then the derivative of their product is negative and

$$
\begin{aligned}
\mid-\int_{0}^{u} \frac{d}{d u}\left(\frac{1}{\phi^{\prime}} \frac{d}{d u}\left(\frac{\phi^{\prime \prime}}{\left(\phi^{\prime}\right)^{2}}\right)\right) e^{\frac{-2 l \phi}{c} d u \mid} & \leq \int_{0}^{u} \frac{d}{d u}\left(\frac{1}{\phi^{\prime}} \frac{d}{d u}\left(\frac{\phi^{\prime \prime}}{\left(\phi^{\prime}\right)^{2}}\right)\right) d u \\
& \leq-\left.\frac{1}{\phi^{\prime}} \frac{d}{d u} \frac{\phi^{\prime \prime}}{\left(\phi^{\prime}\right)^{2}}\right|_{u=0} .
\end{aligned}
$$


Because of the asymptotic exponential decay of $\phi^{\prime \prime}$ a constant $L$ can therefore be prescribed, independent of $\varepsilon$, such that

$$
\begin{gathered}
\left|\left[\frac{\varepsilon}{8 \phi^{\prime}} \frac{d}{d u}\left(\frac{\phi^{\prime \prime}}{\left(\phi^{\prime}\right)^{2}} e\right)^{\frac{-2 u \phi}{\varepsilon}}\right]_{v=0}^{u}-\frac{\varepsilon}{8} \int_{0}^{u} \frac{d}{d u}\left(\frac{1}{\phi^{\prime}} \frac{d}{d u}\left(\frac{\phi^{\prime \prime}}{\left(\phi^{\prime}\right)^{2}}\right)\right) e^{\frac{-2 l \phi}{\varepsilon}} d u\right| \\
\leq \varepsilon L\left(e^{-2 u}+1\right)
\end{gathered}
$$

for all $u>0$.

\section{References}

[1] P. B. Chapman and J. J. Mahony, "Reflection of waves in a slowly varying medium", SIAM J. Appl. Math. 34 (1978) 303-319.

[2] H. and B. S. Jeffreys, Mathematical physics. 3rd Edition (University Press, Cambridge, 1956).

[3] J. Kevorkian, "Perturbation techniques for oscillatory systems with slowly varying coefficients", SIAM Review 29 (1987) 391-460.

[4] N. Krylov and N. N. Bogoliubov, Introduction to nonlinear mechanics (Princeton University Press, Princeton, N. J. 1947).

[5] F. W. J. Olver, Asymptotics and special functions (Academic Press, N.Y., 1974). 